\title{
Asymptotically anti-de Sitter wormholes
}

\author{
Carlos Barceló, ${ }^{1}$ Luis J. Garay, ${ }^{2}$ Pedro F. González-Díaz, ${ }^{3}$ and Guillermo A. Mena Marugán ${ }^{3}$ \\ ${ }^{1}$ Instituto de Astrofísica de Andalucía, CSIC, Camino Bajo de Huétor, 18080 Granada, Spain \\ ${ }^{2}$ Theoretical Physics Group, The Blackett Laboratory, Imperial College, London SW7 2BZ, United Kingdom \\ ${ }^{3}$ Centro de Física “Miguel A. Catalán," Instituto de Matemáticas y Física Fundamental, CSIC, Serrano 121, 28006 Madrid, Spain
}

(Received 24 October 1995)

\begin{abstract}
Starting with a procedure for dealing with general asymptotic behavior, we construct a quantum theory for asymptotically anti-de Sitter wormholes. We follow both the path integral formalism and the algebraic quantization program proposed by Ashtekar. By adding suitable surface terms, the Euclidean action of the asymptoically anti-de Sitter wormholes can be seen to be finite and gauge invariant. This action determines an appropriate variational problem for wormholes. We also obtain the wormhole wave functions of the gravitational model and show that all the physical states of the quantum theory are superpositions of wormhole states.
\end{abstract}

PACS number(s): 04.60.Kz, 04.60.Ds, 04.60.Gw, 98.80.Hw

\section{INTRODUCTION}

Wormholes are topology changes that connect different regions of spacetime which may be far apart [1,2]. In the dilute wormhole approximation $[1,3]$, these regions are regarded as asymptotically large. Wormholes can be represented by quantum states, i.e., solutions of the WheelerDeWitt equation (and the quantum momentum constraints), which satisfy some suitable boundary conditions on the asymptotic regions $[4,5]$. They can also be considered as instantons, solutions of the Euclidean Einstein equations, which join the two asymptotic regions of spacetime by a throat $[2,6,7]$. As saddle points of the Euclidean action, these instantons would allow the Euclidean path integral to be approximated semiclassically, thus representing quantum tunneling effects between the asymptotic regions.

Asymptotically flat wormholes have been extensively studied in the literature [8]. There exist, however, other asymptotic behaviors [7,9-11] that are worth considering. For instance, wormholes whose asymptotic regions are Kantowski-Sachs spacetimes [9], with the topology of $\mathrm{R}^{3} \times S^{1}$, may provide a link between black hole physics and the issue of topology change. Asymptotically anti-de Sitter wormholes are also of particular interest. In this case, the asymptotic regions expand exponentially (in proper time) due to the presence of an effective negative cosmological constant. These wormholes could be regarded as excited states in the sense that the cosmological constant could be interpreted as a nonvanishing asymptotic energy of the matter fields. On the other hand, one should expect that these wormholes could give a nonvanishing contribution to the path integral and, consequently, they should be taken into account in calculations such as those leading to Coleman's mechanism for the vanishing of the effective cosmological constant [3].

It has been argued that wormholes might affect the constants of nature through low energy effective interactions $[2,3,12]$. The existence of a Hilbert structure in the space of wormhole wave functions is essential to turn the apparent nonlocal interaction introduced by wormholes into a local one, as seen from one of the asymptotic regions [2,3,12,13]. Such a Hilbert space structure is therefore necessary in the explicit calculation of these effective interactions.

In this work, we construct the Hilbert space of asymptotically anti-de Sitter wormholes, suggesting a procedure for dealing with other possible asymptotic behavior. We employ the path integral approach to obtain the quantum states and Ashtekar's algebraic program [14] to complete the quantization of these wormholes, including the determination of the physical inner product. Finding a well-defined set of wormhole boundary conditions becomes a central issue in both approaches.

Hawking and Page [4] have proposed that the boundary conditions for the quantum wormhole states should guarantee that their corresponding wave functions are exponentially damped for large three-geometries, so that one recovers the semiclassical behavior expected in the asymptotic limit of large Euclidean configurations. Besides, the wormhole wave functions should be regular for all regular matter fields and three-geometries, including those geometries that degenerate to zero because of an ill-defined slicing of spacetime. From the path integral point of view, these conditions can be accomplished if the wormhole wave functions are defined by the sum over all possible spacetimes with the prescribed asymptotic behavior and over all matter fields that are compatible with the given asymptotic spacetime via the vanishing of the first-class constraints in the asymptotic regions. For instance, if we are dealing with asymptotically flat spacetimes, the energy-momentum tensor of the matter fields will have to vanish at infinite proper time [4,5], or if an asymptotically anti-de Sitter behavior is considered, then the matter content will have to induce an effective negative cosmological constant in the asymptotic region. As a previous step, we implement the wormhole boundary conditions canonically and find an appropriate gauge-invariant action, which is finite for classical wormhole solutions. This amounts to include the surface terms that are characteristic of asymptotic spacetimes (see Refs. $[5,15]$ ) and that remove the infinite contribution of the asymptotic regions.

In order to determine the Hilbert structure of the space of wormholes, and thus reach a consistent quantum theory to describe these states, we follow the algebraic quantization program put forward by Ashtekar [14]. In the following, we briefly summarize the main steps of this program. One must 
first choose a complete set of classical variables that is closed under Poisson brackets and complex conjugation. To each of these elementary variables one associates an abstract operator and constructs the algebra generated by them, imposing on it the canonical commutation relations. One must next find a linear representation of this algebra on a complex vector space and choose explicit operators to represent the firstclass constraints of the system. The subspace annihilated by these constraints supplies the space of quantum states, and the quantum observables are the operators that leave this space invariant [14]. The physical inner product on quantum states can then be determined by requiring that the complex conjugation relations between elementary variables (usually called reality conditions) are realized as Hermitian adjoint relations between quantum observables on the resulting Hilbert space [16]. Actually, if an inner product satisfying this condition exists, it is unique under very general assumptions [17]. The elements in the Hilbert space obtained in this way are the physical states of the theory.

For gravitational systems which exhibit quantum wormhole solutions, if one chooses properly the representation space, it is possible to show that the space of quantum states coincides with that spanned by the wormhole wave functions, provided that the latter is invariant under the action of the quantum observables [18]. Therefore, the inner product of wormholes can in fact be determined by imposing an adequate set of reality conditions, and the corresponding Hilbert space of wormholes can be identified with that of physical states of the quantum theory.

In Sec. II, we present a model which illustrates the general features discussed above. It consists of a scalar field conformally coupled to a homogeneous and isotropic spacetime with a negative cosmological constant. In Sec. III, we show that such a model possesses asymptotically anti-de Sitter wormhole solutions. In Sec. IV, an appropriate action for asymptotically large spacetimes is constructed in the general context of superspace and particularized then to our minisuperspace model. The path integral quantization is discussed in Sec. V. Using the results of this section, we carry out the full algebraic quantization of the model in Sec. VI. We finally summarize and conclude in Sec. VII.

\section{MODEL}

We shall discuss in detail a homogeneous and isotropic gravitational minisuperspace model provided with a conformally coupled scalar field and a negative cosmological constant. As we shall see in Sec. III, this model possesses asymptotically anti-de Sitter instanton solutions.

We start by performing the standard $3+1$ splitting of the Euclidean spacetime metric

$$
d s^{2}=\left(N^{2}+N^{i} N_{i}\right) d \tau^{2}+2 N_{i} d \tau d x^{i}+g_{i j} d x^{i} d x^{j},
$$

where $N$ and $N^{i}$ are the lapse and shift functions and $g_{i j}$ is the metric on the closed three-surfaces of constant time. The Euclidean action can be written in the Hamiltonian form

$$
\tilde{I}=\int d \tau \int d^{3} x\left[\pi^{i j} \dot{g}_{i j}+\pi_{\phi} \dot{\phi}-N \mathscr{H}-N^{i} \mathscr{H}_{i}\right]
$$

in which $\pi^{i j}$ and $\pi_{\phi}$ are the canonical momenta conjugate to the three-metric $g_{i j}$ and the conformal scalar field $\phi$, and the overdot denotes the derivative with respect to the time coordinate $\tau$. In the above expression, $\mathscr{H}$ and $\mathscr{H}_{i}$ are the standard Arnowitt-Deser-Misner (ADM) Hamiltonian and momentum constraints for Euclidean gravity conformally coupled to a scalar field in the presence of a negative cosmological constant $\Lambda$.

The requirements of homogeneity and isotropy, i.e., the restriction to the minisuperspace under consideration, can be imposed by writing the spacetime metric in the form

$$
d s^{2}=\frac{2 G}{3 \pi}\left[N^{2}(\tau) d \tau^{2}+a^{2}(\tau) \Omega_{i j} d x^{i} d x^{j}\right],
$$

$\Omega_{i j}$ being the metric on the unit three-sphere and $G$ Newton's constant; likewise, the scalar field will depend only on the time coordinate, $\phi=\phi(\tau)$. It is convenient to introduce a new variable $\chi$ to describe the conformal scalar field in the following manner:

$$
\phi=\sqrt{\frac{3}{4 \pi G}} \frac{\chi}{a} .
$$

When particularized to this minisuperspace model, the Euclidean action becomes

$$
\tilde{I}=\int d \tau\left[\pi_{a} \dot{a}+\pi_{\chi} \dot{\chi}-N H\right]
$$

Here, $\left(\pi_{a}, \pi_{\chi}\right)$ are the momenta canonically conjugate to the variables $(a, \chi)$, and are related to the superspace canonical momenta $\left(\pi^{i j}, \pi_{\phi}\right)$ through the formulas

$$
\begin{gathered}
\pi^{i j}=\frac{1}{8 \pi G}\left(\frac{\pi_{a}}{a}+\frac{\pi_{\chi} \chi}{a^{2}}\right) \Omega^{i j} \Omega^{1 / 2} \\
\pi_{\phi}=\sqrt{\frac{G}{3 \pi^{3}}} a \pi_{\chi} \Omega^{1 / 2}
\end{gathered}
$$

with $\Omega=\operatorname{det} \Omega_{i j}$. On the other hand, $H$ denotes the Hamiltonian constraint in minisuperspace: namely,

$$
H=\frac{1}{2 a}\left(-\pi_{a}^{2}+a^{2}+\lambda a^{4}+\pi_{\chi}^{2}-\chi^{2}\right),
$$

where $\lambda=-\frac{2 G}{9 \pi} \Lambda>0$.

\section{CLASSICAL SOLUTIONS}

The classical Euclidean solutions of this model can be easily obtained by introducing the conformal time $d \eta=d \tau / a$. If we denote the derivative with respect to this time by a prime, the dynamical equations read

$$
\begin{gathered}
a^{\prime}=-\pi_{a}, \quad \pi_{a}^{\prime}=-a-2 \lambda a^{3}, \\
\chi^{\prime}=\pi_{\chi}, \quad \pi_{\chi}^{\prime}=\chi,
\end{gathered}
$$

while the Hamiltonian constraint is 


$$
\frac{1}{2}\left(-\pi_{a}^{2}+a^{2}+\lambda a^{4}+\pi_{\chi}^{2}-\chi^{2}\right)=0
$$

In the above expressions, we have set the lapse function equal to 1 .

The general solution to Eqs. (3.2) is given by

$$
\chi=A \cosh \eta+B \sinh \eta,
$$

with $A$ and $B$ being two arbitrary real constants. Substituting this solution in the Hamiltonian constraint and using the first equation in (3.1), we get

$$
\left(a^{\prime}\right)^{2}=a^{2}+\lambda a^{4}-2 E,
$$

where $E=\frac{1}{2}\left(A^{2}-B^{2}\right)$. This constraint will have solutions of the wormhole type only if the polynomial that appears on its right-hand side has at least a positive root. This implies that $E$ must be positive. We will restrict ourselves to this case hereafter.

Since $E>0$, we can parametrize the constants $A$ and $B$ as

$$
A=\sqrt{2 E} \cosh \eta_{0}, \quad B=-\sqrt{2 E} \sinh \eta_{0},
$$

with $\eta_{0}$ an arbitrary real parameter. The conformal field $\chi$ can then be rewritten

$$
\chi=\sqrt{2 E} \cosh \left(\eta-\eta_{0}\right) .
$$

In addition, integration of Eq. (3.5) leads to

$$
a(\eta)=a_{M} \operatorname{nc}\left(D^{1 / 4}\left(\eta-\tilde{\eta}_{0}\right) \mid m\right),
$$

where $\operatorname{nc}(u \mid m)$ is the Jacobian elliptic function with parameter $m[19], \tilde{\eta}_{0}$ is a real constant, and

$$
\begin{gathered}
D=1+8 \lambda E, \quad a_{M}=\left(\frac{D^{1 / 2}-1}{2 \lambda}\right)^{1 / 2}, \\
m=\frac{D^{-1 / 2}+1}{2} .
\end{gathered}
$$

One can check that Eqs. (3.1) are then straightforwardly satisfied.

The classical wormhole solutions of the model are therefore parametrized by three independent real constants: $\eta_{0}$, $\tilde{\eta}_{0}$, and $E>0$. Notice that $D>1$ and that $a_{M}$ is the size of the wormhole throat, which coincides with the only positive root of the right-hand side of the constraint (3.5).

It is also possible to obtain the solution to that constraint in terms of the proper time $\tau$. One arrives at the following expression for the scale factor:

$$
a=\frac{1}{\sqrt{2 \lambda}}\left\{D^{1 / 2} \cosh \left[2 \sqrt{\lambda}\left(\tau-\tilde{\tau}_{0}\right)\right]-1\right\}^{1 / 2},
$$

where the new real constant $\tilde{\tau}_{0}$ appears instead of $\tilde{\eta}_{0}$.

Some comments are in order at this point. First, the conformal time $\eta$ tends to a finite value $\eta_{M}$ as the proper time $\tau$ goes to infinity. This is due to the fact that, the scale factor being exponentially large at $\tau \rightarrow \infty$, the integral $\int^{\infty} d \tau / a(\tau)$ converges. This feature is actually reflected by the elliptic function $\operatorname{nc}(u \mid m)$ that describes the scale factor solutions in conformal time, for such a function diverges at the finite point $u=K(m)$, with $K(m)$ being the complete elliptic integral of the first kind [19]. Second, all the solutions that we have obtained have asymptotically anti-de Sitter behavior, as can be easily seen by considering the limit $\tau \rightarrow \infty$ in Eq. (3.11). The globally anti-de Sitter solution corresponds to the limit $D \rightarrow 1$ in that equation. Finally, note that the flat solutions $(\lambda=0)$ cannot be recovered by taking the limit $\lambda \rightarrow 0$. This is not surprising, because the $\lambda$ term in Eq. (3.5) is dominant in the asymptotic region $a \rightarrow \infty$ and therefore provides a singular perturbation to the $\lambda=0$ equations of motion.

\section{SURFACE TERMS}

Action (2.5) is not adequate for studying spacetimes that join onto an asymptotically anti-de Sitter region. Actually, it diverges for classical solutions [10] and can be shown not to be invariant under time reparametrizations that map the initial three-surface onto itself. Moreover, it is not quite clear that this action could correspond then to a variational problem which guaranteed the anti-de Sitter asymptotic behavior of the classical spacetimes. These difficulties can be nonetheless overcome by adding appropriate surface terms to the action. In order to obtain these terms, it appears most convenient to being by considering the general superspace framework, without specializing to any particular asymptotic behavior. We shall then reduce the framework to the homogeneous and isotropic model conformally coupled to a scalar field, discussing first the flat case $\lambda=0$ to circumvent the subtleties that arise when introducing a negative cosmological constant.

\section{A. Superspace}

The gravitational systems under consideration join an initial three-surface onto an asymptotic region. The boundary conditions for the associated variational problem must reflect this fact. The geometry of the initial three-surface and its matter content will be chosen as one of the boundary conditions. The final time boundary conditions must guarantee the prescribed asymptotic behavior (at least for classical solutions). Besides, we would like our system to be invariant under gauge transformations that are not fixed at the final time, so that one can reach a semiclassical picture in which the final surface is not fixed, but asymptotically embedded in a classical spacetime.

Let us assume that the final boundary conditions can be imposed by fixing certain variables $Q^{\alpha}$ at the final time $\tau_{f}$, namely, $\left.Q^{\alpha}\right|_{\tau_{f}}=Q_{f}^{\alpha}$. Notice that the proper time goes to infinity when $\tau \rightarrow \tau_{f}$ for the models studied so far [2,5-7]. In terms of these new variables $Q^{\alpha}$ and their canonically conjugate momenta $P_{\alpha}$ the action (2.2) acquires the form

$$
\begin{aligned}
\tilde{I}= & \int_{0}^{\tau_{f}} d \tau \int d^{3} x\left(P_{\alpha} \dot{Q}^{\alpha}-N \mathscr{H}-N^{i} \mathscr{H}{ }_{i}\right)+\left.\int d^{3} x \mathscr{F}\right|_{\tau_{f}} \\
& -\left.\int d^{3} x \mathscr{F}\right|_{0},
\end{aligned}
$$

where $\mathscr{F}=\mathscr{F}\left[g_{i j}, \phi \mid Q^{\alpha}\right]$ is a generating functional for the 
canonical transformation from the geometrodynamical variables to $\left(Q^{\alpha}, P_{\alpha}\right)$. Then, it can be seen that the action

$$
I=\tilde{I}-\left.\int d^{3} x \cdot \mathscr{F}\right|_{\tau_{f}}
$$

is appropriate for fixing the initial three-geometry, the initial scalar field, and the asymptotic variables $Q^{\alpha}$.

As mentioned above, this action should be invariant under spatial diffeomorphisms and time reparametrizations that are restricted only to map the initial surface $(\tau=0)$ onto itself. These transformations are generated by $\mathscr{H}$ and $\mathscr{H}$ i via the standard Poisson bracket relations $\delta A=\left\{A, \int d^{3} x(\epsilon \mathscr{H}\right.$ $\left.\left.-\epsilon^{i} \mathscr{H}_{i}\right)\right\}$, with $\epsilon$ vanishing at $\tau=0$. The variation of the action $I$ under these transformations is

$$
\delta I=-\left.\int d^{3} x\left(\epsilon \mathscr{H}+\epsilon^{i} \mathscr{H}_{i}-P_{\alpha} \delta Q^{\alpha}\right)\right|_{\tau_{f}},
$$

where we have used the standard gauge variation for the lapse and shift functions [20]. Since the gauge transformations are arbitrary at the final time, the vanishing of the first two terms in the right-hand side of this expression is only ensured by choosing the variables $Q^{\alpha}$ so that the first-class constraints are set to zero in the asymptotic region:

$$
\left.\mathscr{H}\right|_{Q_{f}^{\alpha}}=0,\left.\quad \mathscr{H}_{i}\right|_{Q_{f}^{\alpha}}=0
$$

The values $Q_{f}^{\alpha}$ cannot therefore be fixed in a fully arbitrary way. For the vanishing of the third term in (4.3), on the other hand, we need our canonical coordinates $Q^{\alpha}$ to be locally observable in the asymptotic region, in the sense that the Poisson brackets $\left.\left\{Q^{\alpha}, \mathscr{H}\right\}\right|_{Q_{f}^{\alpha}}$ and $\left.\left\{Q^{\alpha}, \mathscr{H}_{i}\right\}\right|_{Q_{f}^{\alpha}}$ vanish, so that their asymptotic values are left invariant under the gauge transformations of the system.

The resulting action $I$ turns out to be finite for classical solutions under sufficiently general conditions. To see this we first note that, on classical solutions,

$$
\begin{aligned}
I_{\text {class }}= & \int_{0}^{\tau_{1}} d \tau \int d^{3} x\left(\pi^{i j} \dot{g}_{i j}+\pi_{\phi} \dot{\phi}\right)-\left.\int d^{3} x \mathscr{F}\right|_{\tau_{1}} \\
& +\int_{\tau_{1}}^{\tau_{f}} d \tau \int d^{3} x\left(\pi^{i j} \dot{g}_{i j}+\pi_{\phi} \dot{\phi}-\dot{\mathscr{F}}\right),
\end{aligned}
$$

where $\tau_{1}$ is a finite intermediate time. Since the classical solutions should be regular along the entire interval $\left[0, \tau_{1}\right]$ but might blow up asymptotically as $\tau$ approaches $\tau_{f}$, any possible divergence in (4.5) must appear in the last integral. Taking into account the canonical transformation generated by $\mathscr{F}\left[g_{i j}, \phi \mid Q^{\alpha}\right]$, we rewrite this last integral as

$$
\int_{\tau_{1}}^{\tau_{f}} d \tau \int d^{3} x P_{\alpha} \dot{Q}^{\alpha}
$$

If the variables $Q^{\alpha}$ are actually observables, i.e., if their Poisson brackets with the constraints vanish weakly, integral (4.6) vanishes, because these variables are then constant on the classical trajectories. In the more general case in which they are only locally observable at their asymptotic values, $\dot{Q}^{\alpha} \rightarrow 0$ as we approach $\tau_{f}$, and the action will be finite if the term $\int d^{3} x P_{\alpha} \dot{Q}^{\alpha}$ decreases fast enough in the limit $\tau \rightarrow \tau_{f}$. This further restricts the kind of variables that are allowed to be fixed asymptotically.

To summarize, the asymptotic boundary conditions can be canonically implemented by choosing a suitable set of compatible variables and fixing their final values in such a way that they become locally observable. These values must imply, in particular, the asymptotic vanishing of the generators of spatial diffeomorphisms and time reparametrizations. This procedure ensures that the action for the system is gauge invariant, finite, and gives rise to a well-defined variational problem for the boundary conditions under consideration.

\section{B. Asymptotically flat wormholes}

We first consider the case of asymptotically flat spacetimes $(\lambda=0)[5]$ for which action (2.5) can be rewritten as

$$
\tilde{I}=\int_{0}^{\eta_{f}} d \eta\left[\pi_{a} a^{\prime}+\pi_{\chi} \chi^{\prime}-N H\right],
$$

where $\eta$ is again the conformal time, $\eta_{f}=\infty$, and the Hamiltonian constraint $H$ is the difference of the Hamiltonians of two harmonic oscillators, one describing the scale factor and the other the conformal field.

We expect the wormholes solutions of this model to be stationary trajectories of the variational problem with fixed initial values of $a$ and $\chi$ and suitable final values for a complete set of compatible variables which are left invariant under time reparametrizations. These conditions on the variables fixed in the asymptotic region will be clearly satisfied if they are compatible observables of the system.

Given the form of the Hamiltonian constraint, we can choose

$$
E_{a}=\frac{1}{2}\left(a^{2}-\pi_{a}^{2}\right), \quad E_{\chi}=\frac{1}{2}\left(\chi^{2}-\pi_{\chi}^{2}\right)
$$

as our set of compatible observables. The variables

$$
\Theta_{x}=\ln \left(\frac{x+\pi_{x}}{\sqrt{x^{2}-\pi_{x}^{2}}}\right) \quad(x=a, \chi)
$$

are the momenta canonically conjugate to these observables. The canonical transformation from $\left(x, \pi_{x}\right)$ to $\left(E_{x}, \Theta_{x}\right)$ is generated by the function

$$
\begin{aligned}
F_{x}\left(x \mid E_{x}\right) & =-\int_{\sqrt{2 E_{x}}}^{x} d z\left(z^{2}-2 E_{x}\right)^{1 / 2} \\
& =-\frac{x}{2}\left(x^{2}-2 E_{x}\right)^{1 / 2}+E_{x} \ln \left(\frac{x+\sqrt{x^{2}-2 E_{x}}}{\sqrt{2 E_{x}}}\right) .
\end{aligned}
$$

In terms of the new variables, action (4.2) reduces to 


$$
\begin{aligned}
I=\tilde{I}-\left.\left(F_{a}+F_{\chi}\right)\right|_{\eta_{f}}= & \int_{0}^{\eta_{f}} d \eta\left[\Theta_{a} E_{a}^{\prime}+\Theta_{\chi} E_{\chi}^{\prime}-N\left(E_{a}-E_{\chi}\right)\right] \\
& -\left.\left(F_{a}+F_{\chi}\right)\right|_{0},
\end{aligned}
$$

with $\eta_{f}=\infty$. On the other hand, the Hamiltonian constraint $H=E_{a}-E_{\chi}$ generates, via Poisson brackets, the time reparametrizations

$$
\delta E_{x}=\epsilon\left\{E_{x}, H\right\}, \quad \delta \Theta_{x}=\epsilon\left\{\Theta_{x}, H\right\}, \quad \delta N=\epsilon^{\prime},
$$

where the parameter $\epsilon$ depends only on the conformal time. It is then easy to check that the action (4.11), supplemented with the wormhole boundary conditions

$$
E_{a}\left(\eta_{f}\right)=E_{\chi}\left(\eta_{f}\right)=E, \quad \text { with } E>0,
$$

is invariant under time reparametrizations that map the initial surface onto itself [namely, with $\epsilon(0)=0$ ]. The stationary points of this action are the classical trajectories that join an initial three-surface characterized by the scale factor $a(0)=a_{i}$ and the conformal field $\chi(0)=\chi_{i}$ with an asymptotic region in which condition (4.13) is satisfied. This asymptotic condition actually implies that the solutions of the model are asymptotically flat, as can be straightforwardly seen by solving the equation $2 E=a^{2}-\left(a^{\prime}\right)^{2}$. Finally, given the constraint $H=0$ and the dynamical equations $E_{a}^{\prime}=E_{\chi}^{\prime}=0$, the action (4.11) reduces to

$$
I_{\text {class }}=-F_{a}\left(a_{i} \mid E\right)-F_{\chi}\left(\chi_{i} \mid E\right)
$$

on classical solutions. From Eq. (4.10), it then follows that the classical action is always finite provided that $E$ (i.e., the asymptotic energy of the conformal field) is positive.

\section{Asymptotically anti-de Sitter wormholes}

Let us now extend the above analysis to the asymptotically anti-de Sitter case. The situation remains in fact unchanged except in what refers to the scale factor. In the anti-de Sitter case, the part of the Hamiltonian constraint which depends on $a$ and $\pi_{a}$ incorporates a cosmological term, namely, $E_{a}=\frac{1}{2}\left(a^{2}+\lambda a^{4}-\pi_{a}^{2}\right)$. The generating function $F_{a}\left(a \mid E_{a}\right)$ has to be subsequently modified to take care of the nonvanishing cosmological constant. One arrives at

$$
F_{a}\left(a \mid E_{a}\right)=-\int_{a_{M}}^{a} d z\left(z^{2}+\lambda z^{4}-2 E_{a}\right)^{1 / 2},
$$

where $a_{M}$ is the root of the polynomial $a^{2}+\lambda a^{4}-2 E_{a}$ which can be obtained from Eq. (3.9) by substituting $E_{a}$ for E.

Expressions (4.11) and (4.13) still provide the gaugeinvariant action and the boundary conditions for the anti-de Sitter wormholes, respectively. Note, however, that, from our remarks at the end of Sec. III, the final conformal time $\eta_{f}$ will now be finite for all the wormhole solutions of the model. We shall therefore fix $\eta_{f}$ to coincide with the time $\eta_{M}\left(a_{i}, E\right)$ at which the solution (3.8)-(10), verifying $a(0)=a_{i}$, tends to $+\infty$. Finally, one can check that the action on classical solutions again takes the form (4.14), but with $F_{a}\left(a \mid E_{a}\right)$ supplied now by Eq. (4.15).

\section{PATH INTEGRAL}

The path integral which provides the anti-de Sitter quantum wormholes parametrized by the asymptotic value of the conformal field energy $E>0$ is given by

$$
\begin{aligned}
\Psi_{E}\left[a_{i}, \chi_{i}\right]= & \int \mathscr{D} N \mathscr{D} \mu\left(a, \pi_{a}, \chi, \pi_{\chi}\right) \Delta_{\mathrm{FP}} \delta(N-1) \\
& \times \exp \left[-I\left(a, \pi_{a}, \chi, \pi_{\chi}, N\right)\right] .
\end{aligned}
$$

Here, we sum over histories satisfying $a(0)=a_{i}$, $\chi(0)=\chi_{i}$, and $E_{a}\left(\eta_{M}\right)=E_{\chi}\left(\eta_{M}\right)=E$. We recall that $\eta_{M}$ is a constant that depends on the values of $a_{i}$ and $E$. The Faddeev-Popov determinant $\Delta_{\mathrm{FP}}$ can be set equal to the unity, because it does not depend on any of the integration fields for our gauge-fixing condition $N=1$. Integration over $N$ leads then to

$$
\Psi_{E}\left[a_{i}, \chi_{i}\right]=\int \mathscr{D} \mu\left(a, \pi_{a}, \chi, \pi_{\chi}\right) \exp (-I),
$$

where

$$
\begin{aligned}
I= & \int_{0}^{\eta_{M}} d \eta\left[\pi_{a} a^{\prime}+\pi_{\chi} \chi^{\prime}-\frac{N}{2}\left(-\pi_{a}^{2}+a^{2}+\lambda a^{4}+\pi_{\chi}^{2}-\chi^{2}\right)\right] \\
& -\left.\left(F_{a}+F_{\chi}\right)\right|_{\eta_{M} .}
\end{aligned}
$$

The part of this path integral which depends on the conformal field provides the propagator $U\left(E, \eta_{M} \mid \chi_{i}, 0\right)$ of a harmonic oscillator between a fixed initial field $\chi_{i}$ and a constant energy $E_{\chi}=E$ at the final time $\eta_{M}$. With a proper choice of the integration measure, this propagator would be a linear combination of the normalized eigenstates $\varphi_{n}\left(\chi_{i}\right)(n$ $=0,1, \ldots)$ of the harmonic oscillator, namely,

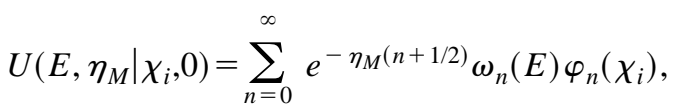

in which $\omega_{n}(E)$ are some coefficients which depend on $E$ and we have set $\hbar=1$. On the other hand, the result of the path integral should satisfy the quantum version of the constraint

$$
-\pi_{\chi}^{2}+\chi^{2}-2 E=0
$$

which, since $E_{\chi}$ is preserved by the dynamics of the system and we have imposed $E_{\chi}=E$ at $\eta_{M}$, holds on all classical trajectories. Therefore using Eq. (5.4), we conclude that $E$ can only take the values $n+\frac{1}{2}$, if the path integral is to be well defined, and then that, up to a global $E$-dependent factor,

$$
U\left(n+\frac{1}{2}, \eta_{M} \mid \chi_{i}, 0\right)=e^{-\eta_{M}(n+1 / 2)} \varphi_{n}\left(\chi_{i}\right) .
$$

Hence, the path integral reduces to

$$
\Psi_{n+\frac{1}{2}}\left[a_{i}, \chi_{i}\right]=\varphi_{n}\left(\chi_{i}\right) \Phi_{n}\left(a_{i}\right),
$$

where 


$$
\begin{aligned}
\Phi_{n}\left(a_{i}\right)= & \int \mathscr{D} \mu\left(a, \pi_{a}\right) \exp \left[-\int_{0}^{\eta_{M}} d \eta\left(\pi_{a} a^{\prime}-\frac{1}{2}\left[-\pi_{a}^{2}+a^{2}\right.\right.\right. \\
& \left.\left.\left.+\lambda a^{4}-(2 n+1)\right]\right)+\left.F_{a}\right|_{\eta_{M}}\right]
\end{aligned}
$$

In this expression, we sum over histories with $a(0)=a_{i}$ and $E_{a}\left(\eta_{M}\right)=n+\frac{1}{2}$. The functions $\Phi_{n}(a)$ must be solutions to the Wheeler-DeWitt equation which follows from the constraint

$$
-\pi_{a}^{2}+a^{2}+\lambda a^{4}-(2 n+1)=0 .
$$

The factor ordering in this Wheeler-DeWitt equation will depend on the integration measure employed in the path integral (5.8). We shall assume a factor ordering of the form

$$
\begin{aligned}
\hat{H}_{a} \Phi_{n}(a) & \equiv \frac{1}{2}\left(-\frac{1}{f(a)} \partial_{a} f(a) \partial_{a}+a^{2}+\lambda a^{4}\right) \Phi_{n}(a) \\
& =\left(n+\frac{1}{2}\right) \Phi_{n}(a),
\end{aligned}
$$

where the function $f(a)$ will be supposed to be analytic and strictly positive at least for $a \geqslant 0$ and such that

$$
\lim _{a \rightarrow \infty} \frac{f^{\prime}(a)}{a^{2} f(a)}=0,
$$

the prime denoting here the first derivative.

If we now restrict our attention to the region $a \in \mathrm{R}^{+}$, so that each different geometry of the type (2.3) is considered only once, it is possible to prove that there actually exists a solution $\Phi_{n}(a)$ to Eq. (5.10) such that it is regular in the positive semiaxis and decreases exponentially for large scale factor. In order to see this, let us consider $\hat{H}_{a}-\left(n+\frac{1}{2}\right)$ as a second order differential operator which annihilates $\Phi_{n}(a)$. The coefficient of $\partial_{a}^{2}$ in this operator is constant. The coefficient of $\partial_{a}$, given by $f^{\prime}(a) / f(a)$, is analytic in $a \geqslant 0$, because $f(a)$ is positive and analytic in this semiaxis. Finally, the nonderivative term is also analytic, as it is a polynomial in $a$. It then follows [21] that, for each fixed $n$, the differential equation (5.10) possesses two linearly independent solutions which are analytic at least for all $a \geqslant 0$. Moreover, provided that condition (5.11) is satisfied, an asymptotic analysis of this differential equation shows that one of these solutions must be exponentially damped in the limit $a \rightarrow \infty$, while the other increases exponentially.

We want to show now that $\Phi_{n}(a)$ should be the exponentially damped solution. For $a_{i} \gg 1$, we expect the semiclassical aproximation to become valid in the path integral, i.e.,

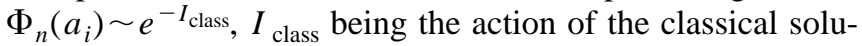
tion to the constraint (5.9) with $a(0)=a_{i}$. For this solution, $a\left(\eta \rightarrow \eta_{M}\right) \rightarrow \infty$ and, admitting that $a^{\prime}=-\pi_{a}$ is positive for $a \gg 1$, one gets

$$
\begin{aligned}
I_{\text {class }} & =-\int_{a_{i}}^{\infty} d z\left[z^{2}+\lambda z^{4}-(2 n+1)\right]^{1 / 2}-\left.F_{a}\right|_{a=\infty} \\
& =\int_{a_{M}}^{a_{i}} d z\left[z^{2}+\lambda z^{4}-(2 n+1)\right]^{1 / 2}
\end{aligned}
$$

where we have substituted Eq. (4.15), and $a_{M}$ is given by Eq. (3.9) with $E=n+\frac{1}{2}$. The integral in the above expresion is positive and diverges in the limit $a_{i} \rightarrow \infty$. As a consequence, the function $\Phi_{n}\left(a_{i}\right)$ is exponentially damped in that limit.

We thus conclude that the functions $\Phi_{n}(a)$, solutions to (5.10) with $n=0,1, \ldots$, satisfy the wormhole boundary conditions if $a$ is restricted to run over the positive axis. Actually, we have shown that these functions are not only regular, but analytic in $a \geqslant 0$.

It is worth remarking that, even though the solutions $\Phi_{n}(a)$ could be analytically extended to the whole real axis, their asymptotic behavior at $a \rightarrow-\infty$ would not be damped unless in exceptional situations, and never for all the functions $\Phi_{n}(a)(n=0,1, \ldots)$, because that would imply that the operator $\hat{H}_{a}$ has exactly the eigenvalue spectrum which characterizes the Hamiltonian of the harmonic oscillator. Therefore, the restriction to $a \in \mathbb{R}^{+}$is essential if we want the wave functions $\Phi_{n}(a)$ to represent quantum wormhole states.

\section{ALGEBRAIC QUANTIZATION}

Our minisuperspace model possesses only one constraint, namely, the Hamiltonian constraint (3.3). To carry out the algebraic quantization, it is convenient to introduce the Lorentzian momenta $\left(\Pi_{a}, \Pi_{\chi}\right)$ canonically conjugate to the scale factor and the conformal field. Then, the Hamiltonian constraint reads

$$
H=\frac{1}{2}\left(\Pi_{a}^{2}+a^{2}+\lambda a^{4}\right)-\frac{1}{2}\left(\Pi_{\chi}^{2}+\chi^{2}\right)=0 .
$$

The symplectic structure on phase space is supplied by the Poisson brackets $\left\{a, \Pi_{a}\right\}=1$ and $\left\{\chi, \Pi_{\chi}\right\}=1$. For Lorentzian geometries and real conformal fields, we have $\chi, \Pi_{a}, \Pi_{\chi} \in \mathbb{R}$. In addition, we shall restrict the scale factor to be positive, $a \in \mathbb{R}^{+}$, so that each different four-geometry is considered only once.

\section{A. Elementary variables}

As pointed out in the Introduction, our first task will consist in choosing a suitable complete set of elementary variables in the phase space of the model. Since the part of the Hamiltonian constraint which depends on the conformal field can be interpreted as the Hamiltonian of a harmonic oscillator, we will describe the degrees of freedom of this field by the annihilation and creation variables

$$
A_{\chi}=\frac{1}{\sqrt{2}}\left(\chi+i \Pi_{\chi}\right), \quad A_{\chi}^{\dagger}=\frac{1}{\sqrt{2}}\left(\chi-i \Pi_{\chi}\right) .
$$

For $\chi, \Pi_{\chi} \in \mathbb{R}$, both $A_{\chi}$ and $A_{\chi}^{\dagger}$ take on all complex values. In addition, $\left\{A_{\chi}, A_{\chi}^{\dagger}\right\}=-i$ and $\bar{A}_{\chi}=A_{\chi}^{\dagger}$, the bar denoting complex conjugation. 
The remaining part of the Hamiltonian constraint,

$$
h=\frac{1}{2}\left(\Pi_{a}^{2}+a^{2}+\lambda a^{4}\right),
$$

can be regarded as the Hamiltonian of a point particle moving on the $a$ axis under the influence of the potential $a^{2}+\lambda a^{4}$. A canonical set of variables in the corresponding phase space is given by $h$ and

$$
\theta=\int_{a_{h}}^{a} d z\left(2 h-z^{2}-\lambda z^{4}\right)^{-1 / 2}=D_{h}^{-1 / 4} \mathrm{cn}^{-1}\left(a_{h}^{-1} a \mid \tilde{m}_{h}\right),
$$

where $\mathrm{cn}^{-1}\left(u \mid \tilde{m}_{h}\right)$ is the inverse Jacobian elliptic function with parameter $\tilde{m}_{h}$ [19], and $D_{h}, a_{h}$, and $m_{h}=1-\tilde{m}_{h}$ are the values taken by the parameters $D, a_{M}$, and $m$ [defined in Eqs. (3.9), (3.10)] when $E=h$. It is not difficult to check that $h$ is the momentum canonically conjugate to $\theta$.

From the above equations, it follows that $h \in \mathbb{R}^{+}$, and that $a_{h}$ is the maximum value permitted classically for $a$ when the energy of the point particle is $h$. On the other hand, taking into account that $\operatorname{nc}(i u \mid m)=\operatorname{cn}(u \mid 1-m)$, Eq. (6.4) can be seen to provide the analytic continuation to the Lorentzian regime of the Euclidean classical solution (3.8), with $h$ and $\theta$ substituting for $E$ and the Lorentzian conformal time, respectively.

Had we neglected the restriction $a \in \mathbb{R}^{+}$, Eq. (6.4) would have implied that, for $h$ fixed, the scale factor should describe orbits in phase space which are periodic in $\theta$, with period

$$
4 \int_{0}^{a_{h}} d z\left(2 h-z^{2}-\lambda z^{4}\right)^{-1 / 2}=4 D_{h}^{-1 / 4} K\left(\tilde{m}_{h}\right),
$$

$K\left(\tilde{m}_{h}\right)$ denoting again the complete elliptic integral of the first kind. However, the restriction to positive scale factors breaks this periodicity, limiting the classical motion in the $\left(a, \Pi_{a}\right)$ plane to only half of each periodic orbit. Since the dynamics is invariant under a flip of sign in $a$, and we have chosen the origin of $\theta$ at the turning point $a_{h}$ of the scale factor, we conlude that all allowed trajectories on phase space can actually be described by letting $h \in \mathbb{R}^{+}$and

$$
\theta \in\left(-I_{h}, I_{h}\right) \quad \text { with } I_{h}=D_{h}^{-1 / 4} K\left(\tilde{m}_{h}\right) .
$$

We can now introduce the annihilationlike and creationlike variables

$$
A_{a}=\sqrt{h} e^{-i \theta}, \quad A_{a}^{\dagger}=\sqrt{h} e^{i \theta} .
$$

These variables verify $\left\{A_{a}, A_{a}^{\dagger}\right\}=-i$ and $\bar{A}_{a}=A_{a}^{\dagger}$. However, given restriction (6.6), their range is not the whole complex plane. Nonetheless, this will not lead to any problem in the quantization of the system, because the only physically relevant conditions on quantum operators reflecting restrictions on the range of classical variables are those which refer to the observables of the quantum theory.

The quotient $A_{a}^{\dagger} / A_{a}=e^{2 i \theta}$ distinguishes all points $\theta$ $\in\left(-I_{h}, I_{h}\right)$ for fixed $h$, because $I_{h}$ can be shown to be within the interval $(0, \pi / 2)$ for positive $h$. As a consequence, expressions (6.7) admit the inversion

$$
h=A_{a}^{\dagger} A_{a}, \quad \theta=-\frac{i}{2} \ln \left(\frac{A_{a}^{\dagger}}{A_{a}}\right) .
$$

The change of variables from $(\theta, h)$ to $\left(A_{a}, A_{a}^{\dagger}\right)$ is therefore analytic in the whole phase space of the model.

In the following, we shall regard $\left(A_{\chi}, A_{\chi}^{\dagger}, A_{a}, A_{a}^{\dagger}\right)$ as our complete set of elementary variables. Notice that this set is indeed closed both under Poisson brackets and complex conjugation.

Let us define now

$$
\begin{gathered}
N_{\chi}=A_{\chi}^{\dagger} A_{\chi}, \quad N_{a}=A_{a}^{\dagger} A_{a}, \\
J_{+}=\frac{1}{\sqrt{2}} A_{\chi}^{\dagger} A_{a}^{\dagger}, \quad J_{-}=\frac{1}{\sqrt{2}} A_{\chi} A_{a} .
\end{gathered}
$$

The Hamiltonian constraint (6.1) can then be rewritten as $H=N_{a}-N_{\chi}=0$. Moreover, taking into account that

$\left\{A_{x}, A_{x}^{\dagger}\right\}=-i, \quad\left\{A_{x}, N_{x}\right\}=-i A_{x}, \quad\left\{A_{x}^{\dagger}, N_{x}\right\}=i A_{x}^{\dagger}$

with $x=\chi, a$, one can check that the variables (6.9), (6.10) are actually observables of the model, because their Poisson brackets with $H$ vanish. Since $N_{\chi}$ and $N_{a}$ coincide modulo the constraint $H=0$, we will restrict all further considerations to the set $\left(J_{+}, J_{-}, N_{\chi}\right)$. This set of observables can be easily proved to be (over)complete.

Given that $A_{\chi}$ and $A_{\chi}^{\dagger}$ can take on any complex value, the range of $J_{+}$and $J_{-}$is the whole complex plane. Besides, recalling that $\bar{A}_{x}=A_{x}^{\dagger}(x=\chi, a)$, we get the reality conditions

$$
\bar{J}_{+}=J_{-}, \quad \bar{N}_{\chi}=N_{\chi} \in \mathbb{R}^{+} .
$$

Finally, we also have

$$
\begin{gathered}
\left\{J_{+}, N_{\chi}\right\}=i J_{+}, \quad\left\{J_{-}, N_{\chi}\right\}=-i J_{-}, \\
\left\{J_{+}, J_{-}\right\}=\frac{i}{2}\left(N_{a}+N_{\chi}\right) \approx i N_{\chi},
\end{gathered}
$$

the last identity holding weakly. Therefore, the observables $\left(J_{+}, J_{-}, N_{\chi}\right)$ generate the Lie algebra of $\mathrm{SO}(2,1)$ under Poisson brackets.

\section{B. Representation space}

In order to quantize the system, we should represent the elementary classical variables of the model via linear operators acting on a certain vector space. The space that we shall choose for this task will be that of complex functions on $\mathbb{R}^{+} \times \mathbb{R}$ spanned by the basis

$$
\psi_{n m}(a, \chi)=\Phi_{n}(a) \varphi_{m}(\chi) \quad\left(a \in \mathbb{R}^{+}, \chi \in \mathbb{R}\right),
$$

with $n$ and $m$ two arbitrary non-negative integers and $\varphi_{m}(\chi)$ the normalized wave functions of the harmonic oscillator. Here, the functions $\Phi_{n}(a)$ are the solutions to Eq. (5.10) which decrease exponentially at infinity. We have shown in Sec. V that these functions are analytic in the semiaxis $a \geqslant 0$. This and the damped asymptotic behavior guarantee that the integrals $\int_{\mathbb{R}^{+}} d a \bar{\Phi}_{n}(a) \Phi_{n}(a)$ converge. We shall 
assume hereafter that the functions $\Phi_{n}(a)$ have been normalized so that the above integrals are equal to the unity.

Our representation space contains all the wormhole solutions constructed in Sec. V, namely, $\psi_{n n}(a, \chi)$. We finally want to show that the basis $\psi_{n m}(a, \chi)$ is linearly independent. Since the wave functions $\varphi_{m}(\chi)$ are known to possess this property, it will suffice to prove the linear independence of the functions $\Phi_{n}(a)$, with $a \in \mathbb{R}^{+}$. Let us then suppose that

$$
\sum_{s=1}^{p} c_{n_{s}} \Phi_{n_{s}}(a)=0
$$

where $\left\{n_{s}\right\}$ is an ordered set of non-negative integers, $p>1$ is another integer, and the $c_{n_{s}}$ 's are complex constants. Acting on both sides of this equation with the operator

$$
\prod_{s=1}^{p-1}\left(\hat{H}_{a}-n_{s}-\frac{1}{2}\right)
$$

in which $\hat{H}_{a}$ is defined in Eq. (5.10), we get

$$
c_{n_{p}}\left(n_{p}-n_{p-1}\right) \cdots\left(n_{p}-n_{1}\right) \Phi_{n_{p}}(a)=0 .
$$

We thus conclude that $c_{n_{p}}$ must vanish, since $\Phi_{n_{p}}(a) \neq 0$ and $n_{p}>n_{s}$ for $s=1, \ldots, p-1$. Substituting now $c_{n_{p}}=0$ in Eq. (6.16) and iterating the above procedure, we arrive at $c_{n}=0$ for all $n \in\left\{n_{s}\right\}$. Therefore, the functions $\Phi_{n}(a)$ on $\mathrm{R}^{+}$are linearly independent, and so is then the basis $\psi_{n n}(a, \chi)$ of our representation space.

\section{Quantization}

The elementary variables $\left(A_{\chi}, A_{\chi}^{\dagger}, A_{a}, A_{a}^{\dagger}\right)$ will now be represented as linear operators on the complex vector space spanned by the functions $\psi_{n m}(a, \chi)$, where $n, m=0,1 \ldots$ The action of the corresponding operators on this basis will be given by

$$
\begin{array}{ll}
\hat{A}_{\chi} \psi_{n m}=\sqrt{m} \psi_{n(m-1)}, & \hat{A}_{\chi}^{\dagger} \psi_{n m}=\sqrt{m+1} \psi_{n(m+1)}, \\
\hat{A}_{a} \psi_{n m}=\sqrt{n} \psi_{(n-1) m}, & \hat{A}_{a}^{\dagger} \psi_{n m}=\sqrt{n+1} \psi_{(n+1) m},
\end{array}
$$

where we have set again $\hbar=1$. Let us also introduce the operators

$$
\hat{N}_{x}=\frac{1}{2}\left(\hat{A}_{x}^{\dagger} \hat{A}_{x}+\hat{A}_{x} \hat{A}_{x}^{\dagger}\right) \quad(x=\chi, a),
$$

to represent the derived classical variables (6.9). From the above definitions, we obtain the nonvanishing commutators

$$
\left[\hat{A}_{x}, \hat{A}_{x}^{\dagger}\right]=\hat{1}, \quad\left[\hat{A}_{x}, \hat{N}_{x}\right]=\hat{A}_{x}, \quad\left[\hat{A}_{x}^{\dagger}, \hat{N}_{x}\right]=-\hat{A}_{x}^{\dagger}
$$

which reproduce the Poisson brackets algebra (6.11) up to the usual factor $i$. Here, $\hat{1}$ is the identity operator.

We shall next represent the Hamiltonian constraint by $\hat{H}=\hat{N}_{a}-\hat{N}_{\chi}$. Recalling that the functions $\psi_{n m}(a, \chi)$ are lin- early independent, it is then straightforward to see that all quantum solutions to the Hamiltonian constraint have the form

$$
\Psi(a, \chi)=\sum_{n=0}^{\infty} c_{n} \psi_{n n}(a, \chi)
$$

where the $c_{n}$ 's are arbitrary complex numbers. The vector space of quantum states, $V_{p}$, is thus spanned by the wormhole wave functions $\psi_{n n}(a, \chi)$.

Defining

$$
\hat{J}_{+}=\frac{1}{\sqrt{2}} \hat{A}_{\chi}^{\dagger} \hat{A}_{a}^{\dagger}, \quad \hat{J}_{-}=\frac{1}{\sqrt{2}} \hat{A}_{\chi} \hat{A}_{a},
$$

we get, from Eqs. (6.19) and (6.20),

$$
\begin{gathered}
\hat{J}_{+} \psi_{n n}=\frac{1}{\sqrt{2}}(n+1) \psi_{(n+1)(n+1)}, \\
\hat{J}_{-} \psi_{n n}=\frac{1}{\sqrt{2}} n \psi_{(n-1)(n-1)}, \\
\hat{N}_{\chi} \psi_{n n}=\left(n+\frac{1}{2}\right) \psi_{n n}=\hat{N}_{a} \psi_{n n} .
\end{gathered}
$$

The above operators are hence quantum observables, for they leave the space $V_{p}$ of quantum states invariant.

Notice that $\hat{N}_{\chi}$ and $\hat{N}_{a}$ coincide on $V_{p}$ due to the Hamiltonian constraint. On the other hand, comparison of Eqs. (6.10) and (6.24) shows that $\hat{J}_{+}$and $\hat{J}_{-}$represent the classical observables $J_{+}$and $J_{-}$. We also have, on $V_{p}$,

$$
\left[\hat{J}_{+}, \hat{J}_{-}\right]=-\hat{N}_{\chi}, \quad\left[\hat{J}_{+}, \hat{N}_{\chi}\right]=-\hat{J}_{+}, \quad\left[\hat{J}_{-}, \hat{N}_{\chi}\right]=\hat{J}_{-},
$$

which is the algebra of commutators that follows from the corresponding Poisson brackets. The vector space $V_{p}$ carries then a linear representation of the algebra of physical observables of the model, namely, the Lie algebra of $\mathrm{SO}(2,1)$. This representation is actually irreducible, because all the elements in the basis $\psi_{n n}(a, \chi)$ of $V_{p}$ can be reached from each other through the repeated action of the observables $\hat{J}_{+}$and $\hat{J}_{-}$.

To determine the inner product on $V_{p}$, we must impose the reality conditions (6.12) as adjointness relations between quantum observables, i.e., $\hat{J}_{+}^{\star}=\hat{J}_{-}$and $\hat{N}_{\chi}^{\star}=\hat{N}_{\chi}$ (the star denoting the Hermitian adjoint). In addition, since $N_{\chi} \in \mathrm{R}^{+}$, the operator $\hat{N}_{\chi}$ should be positive on the resulting Hilbert space of physical states. In fact, the relation $\hat{J}_{+}^{\star}=\hat{J}_{-}$suffices to fix the following inner product on $V_{p}$, up to a positive constant factor:

$$
\langle\Gamma, \Psi\rangle=\left\langle\sum_{m=0}^{\infty} d_{m} \psi_{m m}, \sum_{n=0}^{\infty} c_{n} \psi_{n n}\right\rangle=\sum_{n=0}^{\infty} \bar{d}_{n} c_{n},
$$

where we have made use of expression (6.23), valid for all quantum states. 
The completion of the vector space $V_{p}$ with respect to the above product supplies then the physical Hilbert space $\mathscr{H}_{p}$ of the quantum theory. It is clear from Eq. (6.29) that $\mathscr{H}_{p}$ is isomorphic to $l^{2}$, the space of square summable sequences. One can also easily check that the observable $\hat{N}_{\chi}$ is indeed a positive operator on $\mathscr{H}_{p}$. So all the reality conditions on the observables of the system have been satisfactorily dealt with.

It is worth pointing out that, $V_{p}$ being spanned by the wormhole wave functions $\psi_{n n}(a, \chi)$, every physical state in the Hilbert space $\mathscr{H}_{p}$ can be interpreted as a superposition of quantum wormholes. The inner product (6.29) can then be regarded as the one picked out on the space of wormholes by the reality conditions.

To close this section, we shall prove that the product obtained on $V_{p}$ can be equivalently written in the form

$$
\langle\Gamma, \Psi\rangle=\int_{\mathrm{R}^{+}} d a \int_{\mathrm{R}} d \chi \bar{\Gamma}(a, \chi) \Psi(a, \chi) .
$$

Given that the eigenstates $\varphi_{n}(\chi)$ of the harmonic oscillator form an orthonormal basis of $L^{2}(\mathbb{R}, d \chi)$ and that the functions $\Phi_{n}(a)$ have been chosen to have unit norm in $L^{2}\left(\mathbb{R}^{+}, d a\right)$, we get

$$
\begin{array}{r}
\sum_{m=0}^{\infty} \bar{d}_{m} \sum_{n=0}^{\infty} c_{n} \int_{\mathbb{R}^{+}} d a \bar{\Phi}_{m}(a) \Phi_{n}(a) \int_{\mathbb{R}} d \chi \bar{\varphi}_{m}(\chi) \varphi_{n}(\chi) \\
=\sum_{n=0}^{\infty} \bar{d}_{n} c_{n},
\end{array}
$$

from what it follows that the right-hand sides of Eqs. (6.29) and (6.30) actually coincide on $V_{p}$.

\section{CONCLUSIONS}

Among the topology changes that may take place in asymptotically large regions, the study of tunneling effects mediated by wormholes in asymptotically anti-de Sitter regions of the universe, in which the effective cosmological constant is negative is of particular interest in cosmology. It did not seem quite clear whether these tunnelings could be consistently described quantum mechanically or, at least, semiclassically. In this work, we have shown that it is actually possible to construct a quantum theory for this kind of topology changes, at least at the level of a minisuperspace model.

We have considered a homogeneous and isotropic minisuperspace model with a negative cosmological constant and a conformally coupled massless scalar field. The classical solutions to the Euclidean equations of motion and the Hamiltonian constraint are asymptotically anti-de Sitter wormholes. Such solutions are parametrized by three arbitrary constants that account for the initial scale factor and conformal field as well as for the energy of the conformal field, which must be positive.

Starting with a general analysis in superspace, we have seen that adding suitable surface terms renders the Euclidean action finite on classical solutions, while ensuring its gauge invariance and determining a well-defined variational problem consistent with appropriate wormhole boundary conditions. For our minisuperspace model, these boundary conditions essentially amount to identifying the gravitational and conformal field energies with an equal fixed value in the asymptotically anti-de Sitter region. Since the obtained action is finite on classical solutions, it could be used to reach a consistent semiclassical treatment for the asymptotically anti-de Sitter wormholes.

Two procedures have been employed in order to quantize our minisuperspace model. We have first written the path integral in terms of our Euclidean action. We have argued that wormhole wave functions can be obtained from this path integral as the product of an eigenfunction of the harmonic oscillator for the conformal field and a wave function for a scale factor restricted to be positive.

To carry out a thorough and complete quantization of the system we have then followed Ashtekar's program. Thus, we have represented an appropriately chosen set of elementary variables as quantum operators acting on a vector space of functions which contains the wormhole solutions of the model. The Lorentzian reality conditions have then enabled us to determine the physical inner product. This can be understood as an inner product in the space of quantum wormholes. All the wormhole wave functions turn out to have finite norm and, moreover, provide an orthonormal basis of the space of physical states.

\section{ACKNOWLEDGMENTS}

We are very grateful to Mariano Moles for helpful discussions and support. C.B. was supported by a Spanish Ministry of Education and Science (MEC) grant. L.J.G. was supported by the MEC and the British Council. P.G.-D. acknowledges DGICYT for financial support under Research Projects Nos. PB94-0107 and PB93-0139. G.A.M.M. was supported by funds provided by DGICYT and MEC under Contract Adjunct to the Project No. PB93-0139.
[1] A. Strominger, in Particles, Strings and Supernovae, edited by A. Jevicki and C. I. Tan (World Scientific, Singapore, 1989); S. W. Hawking, Mod. Phys. Lett. A 5, 145 (1990); 5453 (1990).

[2] S. W. Hawking, Phys. Lett. B 195, 337 (1987); Phys. Rev. D 37, 904 (1988).

[3] S. Coleman, Nucl. Phys. B310, 643 (1988); I. Klebanov, L. Susskind, and T. Banks, ibid. B317, 665 (1989); S. W. Hawking, ibid. B335, 155 (1990).

[4] S. W. Hawking and D. N. Page, Phys. Rev. D 42, 2655 (1990).
[5] L. J. Garay, Phys. Rev. D 44, 1059 (1991).

[6] S. Giddings and A. Strominger, Nucl. Phys. B306, 890 (1988).

[7] J. J. Halliwell and R. Laflamme, Class. Quantum Grav. 6, 1839 (1989).

[8] See, for instance, L. J. Garay, Ph.D. thesis, Universidad Autónoma de Madrid, 1992, and references therein.

[9] L. M. Campbell and L. J. Garay, Phys. Lett. B 254, 49 (1991).

[10] R. C. Myers, Phys. Rev. D 38, 1327 (1988).

[11] J. E. Lidsey, Phys. Lett. B 352, 207 (1995). 
[12] S. Coleman, Nucl. Phys. B307, 867 (1988); L. J. Garay and J. García-Bellido, ibid. B400, 416 (1993).

[13] S. Giddings and A. Strominger, Nucl. Phys. B307, 854 (1988).

[14] A. Ashtekar, in Lectures on Non-Perturbative Canonical Gravity, edited by L. Z. Fang and R. Ruffini (World Scientific, Singapore, 1991); A. Ashtekar and R. S. Tate, J. Math. Phys. 35, 6434 (1994).

[15] G. W. Gibbons, S. W. Hawking, and M. Perry, Nucl. Phys. B138, 141 (1978); S. W. Hawking and G. T. Horowitz, Report No. DAMTP-R-94-52, gr-qc/9501014 (unpublished).

[16] For a discussion of the procedure to determine the physical inner product, see G. A. Mena Marugán, J. Math. Phys. 37,
196 (1996).

[17] A. Rendall, Class. Quantum Grav. 10, 2261 (1993); report, gr-qc/9403001 (erratum) (unpublished).

[18] G. A. Mena Marugán, Class. Quantum Grav. 11, 2205 (1994); Phys. Rev. D 50, 3923 (1994).

[19] Handbook of Mathematical Functions, Natl. Bur. Stand. Appl. Math. Ser. No. 55, revised 9th ed., edited by M. Abramowitz and I. A. Stegun (U.S. GPO, Washington, D.C., 1970).

[20] C. Teitelboim, Phys. Rev. D 25, 3159 (1982).

[21] See, for instance, C. M. Bender and S. A. Orszag, Advanced Mathematical Methods for Scientists and Engineers, 2nd ed. (McGraw-Hill, Singapore, 1984). 